Gynäk. Rdsch. 1988;28(suppl. 1):I-III

\title{
Contents, Vol. 28, Supplement 1, 1988
}

Inhalt

Laudatio

Dreher, E 1

Senologie heute

Spreng, M 3

Probleme beim Vorliegen radiologisch erkannter, nichttastbarer

Mammaveränderungen

BrundelRe, R 11

Lightscanning. Stellenwert in der Diagnostik des Mammakarzinoms

Marques, L.B 20

Chirurgische Therapie des Mammakarzinoms

Dreher, E 27

Rekonstruktion der weiblichen Brust nach Mastektomie

Tschopp, $\mathrm{H} \quad 36$

Präkanzerose der Mamma

Rageth, S 57

Adjuvante Behandlung des Mammakarzinoms

Goldhirsch, A.; Castiglione, $M \quad 65$

Fragen zur Hormon- und Chemotherapie beim metastasierenden

Mammakarzinom

Brunner,K, W 76

Radioonkologische Therapie des Mammakarzinoms

Lampret, T.; Veraguth, P.C 84 Festschrift für Max Berger

Bernisches Mammasymposivm

Festschrift für Max Berger

Bernisches Mammasymposium

Bern, 22. September 1987

Herausgeber: E. Dreher, Bern

11 Abbildungen und 25 Tabellen, 1988

ícíp m

Gynäkologische Rundschau Vol. 28, Supplementum 1,1988

S. Karger · Verlag fur Medizin und Naturwissenschaften

Basel · München · Paris · London · New York · New Delhi · Singapore · Tokyo · Sydney Alle Rechte vorbehalten.

Ohne schriftliche Genehmigung des Verlags dürfen diese Publikation oder Teile daraus nicht in andere Sprachen übersetzt oder in irgendeiner Form mit mechanischen oder elektronischen Mitteln (einschliesslich Fotokopie, Tonaufnahme und Mikrokopie) reproduziert oder auf einem Datenträger oder einem Computersystem gespeichert werden. 
(C) Copyright 1988 by S. Karger AG, Postfach, CH- 4009 Basel (Schweiz) Printed in Switzerland by Thür AG Offsetdruck, Pratteln ISBN 3-8055-4832-X 